\title{
The Influence of Sex, Ethnicity, and CYP2B6 Genotype on Bupropion Metabolism as an Index of Hepatic CYP2B6 Activity in Humans
}

\author{
Katarina Ilic, ${ }^{1}$ Roy L. Hawke, Ranjit K. Thirumaran, Erin G. Schuetz, J. Heyward Hull, Angela D. \\ M. Kashuba, Paul W. Stewart, Celeste M. Lindley, and Mei-Ling Chen \\ Division of Pharmacotherapy and Experimental Therapeutics, UNC Eshelman School of Pharmacy (K.I., R.L.H., J.H.H., \\ A.D.M.K., C.M.L), Biostatistics Department, School of Public Health (P.W.S.), University of North Carolina, Chapel Hill, \\ North Carolina; University of Belgrade, School of Pharmacy, Belgrade, Serbia (K.I.); Department of Pharmaceutical \\ Sciences, St. Jude Children's Research Hospital, Memphis, Tennessee (R.K.T., E.G.S.); and Office of Pharmaceutical \\ Science, Center for Drug Evaluation and Research, Food and Drug Administration, Silver Spring, Maryland (M.L.C.)
}

Received July 31, 2012; accepted December 13, 2012

\begin{abstract}
The effects of sex, ethnicity, and genetic polymorphism on hepatic CYP2B6 (cytochrome P450 2B6) expression and activity were previously demonstrated in vitro. Race/ethnic differences in CYP2B6 genotype and phenotype were observed only in women. To identify important covariates associated with interindividual variation in CYP2B6 activity in vivo, we evaluated these effects in healthy volunteers using bupropion (Wellbutrin SR GlaxoSmithKline, Research Triangle Park, NC) as a CYP2B6 probe substrate. A fixed 150$\mathrm{mg}$ oral sustained-release dose of bupropion was administered to 100 healthy volunteers comprising four sex/ethnicity cohorts $(n=25$ each): Caucasian men and Caucasian, African American, and Hispanic women. Blood samples were obtained at 0 and 6 hours postdose for the measurement of serum bupropion (BU) and hydroxybupropion (HB) concentrations. Whole blood was obtained at baseline for CYP2B6 genotyping. To characterize the relationship
\end{abstract}

between CYP2B6 activity and ethnicity, sex, and genotype when accounting for serum BU concentrations (dose-adjusted $\log _{10^{-}}$ transformed), analysis of covariance model was fitted in which the dependent variable was CYP2B6 activity represented as the $\log _{10^{-}}$ transformed, metabolic ratio of $\mathrm{HB}$ to BU concentrations. Several CYP2B6 polymorphisms were associated with CYP2B6 activity. Evidence of dependence of CYP2B6 activity on ethnicity or genotypeby-ethnicity interactions was not detected in women. These results suggest that CYP2B6 genotype is the most important patient variable for predicting the level of CYP2B6 activity in women, when measured by the metabolism of bupropion. The bupropion metabolic ratio appears to detect known differences in CYP2B6 activity associated with genetic polymorphism, across different ethnic groups. Prospective studies will be needed to validate the use of bupropion as a probe substrate for clinical use.
Introduction

Cytochrome P450 2B6 (CYP2B6) represents approximately 5\% of the total liver microsomal CYP content (Gervot et al., 1999; Stresser and Kupfer 1999). Its expression and enzyme activity has been shown to vary approximately 100-fold in human liver (Faucette et al., 2000).

This work was supported by the Office of Women's Health, U.S. Food and Drug Administration [Grant FRP\#: FDA-SOL-05-00050]. K. I. was a drug research and development fellow (2006-2008) supported by GlaxoSmithKline.

${ }^{1}$ Current affiliation: School of Pharmacy, University of Belgrade, Belgrade, Serbia.

Parts of the data in this article were presented at the American Society for Clinical Pharmacology and Therapeutics Annual Meeting; 2-5 April 2008; Orlando, FL. [published in abstract form: Ilic K, Hogeland G, Rezk N, White N, Lamba J, Lee C, Kasuba ADM, Schuetz EG, Lindley CM, Chen ML, and Hawke RL (2008) The influence of sex, race/ethnicity and CYP2B6 genotype on bupropion metabolism. Clin Pharm Ther 83:81].

dx.doi.org/10.1124/dmd.112.048108
CYP2B6 is also expressed in extrahepatic tissues such as brain, kidney, intestine (Gervot et al., 1999), uterine endometrium (Hukkanen et al., 1998), skin (Janmohamed et al., 2001), lung (Ding and Kaminsky, 2003), and heart (Thum and Borlak, 2000). CYP2B6 can partially or completely metabolize approximately 70 substrates (Ekins et al., 1998; Ekins and Wrighton, 1999). It has been shown that CYP2B6 contributes to the metabolism of the anticancer pro-drugs cyclophosphamide (Chang et al., 1993) and ifosphamide (Roy et al., 1999) and to the nonnucleosidic reverse transcriptase inhibitors such as efavirenz (Ward et al., 2003) and nevirapine (Erikson et al., 1999).

CYP2B6 is one of the most polymorphic cytochrome P450 (P450) genes in humans and currently has 30 defined alleles with over 100 described polymorphisms (http://www.imm.ki.se/CYPalleles/cyp2b6. htm). CYP2B6 gene polymorphism includes copy number variants, missense mutations, insertions and deletions, and mutations affecting gene expression and activity. The polymorphic nature of the cytochrome P450 (CYP450) genes affects individual drug response and adverse reactions to a great extent (Ingelman-Sundberg et al., 2007). Studies of

ABBREVIATIONS: AAW, African-American women; ANCOVA, analysis of covariance; AUC, area under the curve; BMI, body mass index; BU, bupropion; CAW, Caucasian-American women; CAM, Caucasian-American men; CYP2B6, cytochrome P450 2B6; HAW, Hispanic-American women; HB, hydroxybupropion; MR, metabolic ratio; P450, cytochrome P450; PCR, polymerase chain reaction; SNP, single nucleotide polymorphisms. 
human liver tissues have shown that women tend to express higher levels of CYP2B6 in liver than men (Lamba et al., 2003). Race/ethnic differences in CYP2B6 genotype and phenotype were observed in women, but not in men. The CYP2B6 activity was 3.6- and 5.0-fold higher, respectively, in Hispanic women than in Caucasian $(P<0.022)$ and African-American $(P<0.038)$ women. The level of CYP2B6 expression has also been linked to genetic polymorphism. Single nucleotide polymorphisms (SNPs) in intron-3 break point $15582 \mathrm{C}>\mathrm{T}$ (which is frequently in combination with the exon-4 516G $>\mathrm{T}$ and influences CYP2B6 alternative splicing) and the exon-9 1459C $>$ T SNP were reported to result in low CYP2B6 activity in women but not men (Lamba et al., 2003).

Several probe substrates have become extremely useful in vitro and in vivo for evaluating cytochrome P450 activity (Tucker et al., 1998; Streetman et al., 2000). However, only two CYP2B6 substrates have emerged as potential probe substrate for quantifying the level of CYP2B6 activity in humans. In vitro investigations using human liver microsomes and recombinant P450s suggest that CYP2B6 is the primary $\mathrm{P} 450$ enzyme catalyzing the metabolism of bupropion (Faucette et al., 2000; Hesse et al., 2000) and efavirenz (Ward et al., 2003).

Stereoselective bupropion hydroxylation has been used as in vivo phenotypic probe for CYP2B6 activity (Kharasch et al., 2008) and to investigate potential CYP2B6 drug interactions in in vivo pharmacokinetic studies (Hogeland et al., 2007). It has been shown that CYP2B6 polymorphisms influence the pharmacokinetics of bupropion (Kirchheiner et al., 2003). However, reports of the effects of variant alleles on bupropion's pharmacokinetics and clinical outcomes are conflicting and the relationship between its pharmacokinetics and CYP2B6 activity has not been firmly established in vivo. In contrast, $C Y P 2 B 6$ polymorphisms could influence metabolism of efavirenz and consequently be associated with differences in clinical outcomes (Gounden et al., 2010). Thus efavirenz might be a useful CYP2B6 probe substrate in vivo. However, evidence of important drug-drug interactions between efavirenz and other CYP2B6 substrates is limited, and practical use of efavirenz as a probe substrate is restricted by concerns regarding human immunodeficiency virus resistance in individuals for whom HIV status is not known.

In this cohort-comparison study, we investigated the relationship between bupropion metabolism and sex, ethnicity, and CYP2B6 genetic polymorphism. Four cohorts of normal healthy adult volunteers were studied: Caucasian-American men (CAM), Caucasian-American women (CAW), Hispanic-American women (HAW), and African-American women (AAW). The polymorphisms investigated were defined by single nucleotide polymorphisms known to alter CYP2B6 function.

\section{Materials and Methods}

Study Design. All subjects in the four cohorts received a fixed open-label dose of bupropion (BU) as a probe substrate for the determination of CYP2B6 activity. Activity was measured as the $\log _{10}$-transformed metabolic ratio of hydroxybupropion (HB) to BU concentrations in blood drawn 6 hours postdose. The single time point concentration ratio method was supported by an analysis of pharmacokinetic data from a previous BU study with 12 healthy volunteers (Hogeland et al., 2007) in which serial blood concentrations suggested that at time points between 6 and 48 hours, $\log _{10}(\mathrm{HB})$ and $\log _{10}(\mathrm{HB} /$ $\mathrm{BU})$ concentrations were correlated with $\log _{10}[\mathrm{HB}$ AUC(area under the curve $\left.)_{-\infty-\infty}\right]$ and $\log _{10}\left[\left(\mathrm{HB} \mathrm{AUC} \mathrm{C}_{\mathrm{o}-\infty}\right) /\left(\mathrm{BU} \mathrm{AUC}_{\mathrm{o}-\infty}\right)\right]$, respectively. Upon analysis, the 6-hour time point was chosen because of its high correlation (correlation coefficient $0.91, R^{2}=0.76$ ) between the concentration ratio and AUC ratio (unpublished data). Each of the four cohorts comprised 25 healthy adult volunteers of ages 18-50 years. All subject groups studied lived in the same area. Each subject had reported that their four grandparents shared a common race/ethnicity. Eligibility did not depend on height, weight, or body mass index (BMI). Screening for eligibility was based on self-reported ethnicity, medical history, screening physical examination and interview, vital signs, and clinical laboratory measurements [sodium, potassium, chloride, bicarbonate, the blood urea nitrogen test, creatinine, glucose, calcium, magnesium, phosphorous, total bilirubin, total protein, albumin, aspartate aminotransferase, alanine aminotransferase, alkaline phosphatase, gamma-glutamyl transpeptidase, and lactate dehydrogenase, coagulation panel (prothrombin time/ partial thromboplastin time), and urinalysis]. Exclusion criteria included the following: ongoing medical illness; allergy to BU; history of seizures or gastrointestinal/ hepatobiliary surgery; reported history of alcohol abuse, smoking, illicit drug use; use of prescription medication within the past month or grapefruit juice and over-the-counter medication during the last two weeks; use of oral contraceptives or lactating or pregnant. Any laboratory value clinically significantly outside the reference ranges previously established by the McLendon Laboratories of the University of North Carolina Hospitals was also grounds for exclusion.

The study was conducted at the Verne S. Caviness General Clinical Research Center at the University of North Carolina (UNC) at Chapel Hill from July 1, 2006 to November 6, 2007. Subjects arrived after an overnight fast. Women of child bearing potential had a urine pregnancy test prior to dosing performed at McClendon UNC Hospital Laboratory. Each subject received a fixed sustained-release oral dose of $150 \mathrm{mg}$ bupropion (GlaxoSmithKline; Lot\# 6ZP2500), along with $240 \mathrm{ml}$ of water. In this case subjects received a fixed amount $(150 \mathrm{mg}$ ) rather than the same dosage of BU. This necessitated a weight-adjusted dose normalization of plasma concentrations before statistical analysis, because subjects received different BU dosage calculated on a per kilogram body weight basis. All subjects were instructed not to eat and drink alcoholic or caffeine-containing beverages until the 6-hour blood sample was drawn. However, drinking water was allowed.

The study protocol and subject-informed consent were approved by The University of North Carolina Institutional Review Board and Research Involving Human Subjects Committee of the Food and Drug Administration. The study has been carried out in accordance with The Declaration of Helsinki. Written consent was obtained from each participant.

Blood Sample Collection. For BU and HB quantification, blood was taken prior to BU dosing and 6 hours postdose. A 7-ml sample of blood was drawn from a forearm vein into sterile sodium EDTA tubes. Upon collection, tubes were placed on ice for 30 minutes and plasma was separated by centrifugation at $3000 \mathrm{rpm}$ for 15 minutes at $-4^{\circ} \mathrm{C}$. Plasma was then aliquoted into cryovials and stored at $-80^{\circ} \mathrm{C}$ until analysis. For genotyping of $C Y P 2 B 6$, blood was collected prior to BU administration.

CYP2B6 Genotyping. Genomic DNA was extracted from the peripheral whole blood of each subject by use of a DNA extraction kit (Qiagen, Valencia, CA). CYP2B6 SNPs at intron 3 branch point $(15582 \mathrm{C}>\mathrm{T})$, exon $4(516 \mathrm{G}>\mathrm{T}$; Q172H, rs3745274), exon 5 (785A $>$ G; K262R, rs22794343), exon 7 (983T $>$ C, I328T, rs2399499), and exon 9 (1459 C > T, R487C, rs3211371) were genotyped by resequencing. Respective exons were amplified using intronic primers and the polymerase chain reaction (PCR) conditions described earlier (Lambda et al., 2003). Prior to sequencing, unincorporated nucleotides and primers were removed by incubation with shrimp alkaline phosphatase (USB; Cleveland, $\mathrm{OH}$ ) and exonuclease I (USB) for 30 minutes at $37^{\circ} \mathrm{C}$, followed by enzyme inactivation at $80^{\circ} \mathrm{C}$ for 15 minutes. Sequencing was carried out with an ABI Prism 3700 Automated Sequencer using the PCR primers or internal primers (sequence available on request). Sequences were assembled using the PhredPhrap-Consed package (University of Washington; Seattle, WA; http://droog. mbt.washington.edu/PolyPhred.html), which automatically detects the presence of heterozygous single nucleotide substitutions by fluorescence-based sequencing of PCR products (Nickerson et al., 1997).

BU and HB Assays. BU and HB plasma concentrations were quantified using an Agilent 1100 Series liquid chromatography-mass spectrometry (Agilent Technologies, Santa Clara, CA) with positive electrospray ionization. Blood plasma samples obtained from subjects 6 hours after bupropion administration were thawed, and $10 \mu \mathrm{l}$ of $4 \mu \mathrm{g} / \mathrm{ml}$ tripolidine (internal standard) was added to $250 \mu \mathrm{l}$ of the sample. Chromatographic separation was carried out on a Phenomenex C8 $(3.2 \times 100 \mathrm{~mm}, 3.0 \mu \mathrm{m})$ column with gradient elution ( $4 \%$ to $50 \%$ acetonitrile in $5 \mathrm{mM}$ ammonium acetate, $\mathrm{pH} 4.6$ ) at a flow rate of $0.3 \mathrm{ml} / \mathrm{min}$. All analytes were converted to free bases by addition of $100 \mu \mathrm{l}$ of $0.1 \mathrm{~N}$ potassium hydroxide. Liquid-liquid phase 
extraction was performed with $1.5 \mathrm{ml}$ hexane:isoamyl alcohol (96:4). The organic layer was collected, and $50 \mu \mathrm{l}$ of $4 \mathrm{~N}$ hydrochloric acid was added to each sample. The eluted solutions were taken to dryness in a Turbo Vap LV evaporator (Zymark Corporation, Hopkinton, MA), then the residues were reconstituted in $150 \mu \mathrm{l}$ of the mobile phase and $25 \mu \mathrm{l}$ of sample solution was injected into Agilent 1100 Series liquid chromatography-massspectrometer. Liquid chromatography-mass spectrometry analyses were carried out with the use of positive electrospray ionization. Chromatographic separation was performed on a Phenomenex C-8 $(3.2 \times 100 \mathrm{~mm}, 3.0 \mu \mathrm{m})$ column with the use of gradient elution with a flow rate of $0.3 \mathrm{ml} / \mathrm{min}$. Mobile phase A consisted of $4 \%$ acetonitrile and $96 \%$ water containing $5 \mathrm{mM}$ ammonium acetate at $\mathrm{pH} 4.6$. Mobile phase B consisted of $50 \%$ acetonitrile and $50 \%$ water containing $5 \mathrm{mM}$ ammonium acetate at $\mathrm{pH}$ 4.6. The lower limit of quantification was $1 \mathrm{ng} / \mathrm{ml}$ for both analytes. Inter-and intraday coefficients of variation were $\leq 10 \%$. The analytical precision of each series was controlled by including two standard solutions spiked with 20 and $100 \mathrm{ng} / \mathrm{ml}$ of bupropion and 50 and $200 \mathrm{ng} / \mathrm{ml}$ hydroxibupropion, respectively. Buproprion was purchased from Sigma, (St. Louis, MO) and hydroxybuproprion was purchased from Gentest, BD Bioscience (Woburn, MA).

Data Analysis Strategy. Descriptive statistical methods were used to compare the four cohorts in terms of age (years), height $(\mathrm{cm})$, weight $(\mathrm{kg})$, and body mass index $\left(\mathrm{kg} / \mathrm{m}^{2}\right)$. At the time of the study, BU was only available in a fixed, $150 \mathrm{mg}$ sustained-release dosage form. Therefore, subjects could not receive a dose of $\mathrm{BU}$ adjusted for differences in body weight. Without normalizing BU plasma concentrations for differences in the actual dose per kilogram body weight each subject received, BU plasma concentrations would vary inversely to body weight and volume of distribution. Accordingly, prior to analysis, all 6-hour BU and $\mathrm{HB}$ concentrations were adjusted to correspond to a standard dose for a 70-kg weight individual. These dose-adjusted (normalized) $\mathrm{BU}$ and $\mathrm{HB}$ concentrations were then transformed to $\log _{10}$ scale because previous studies had suggested that these concentrations arise from underlying population distributions that are approximately log-normal (Julious and Debarnot, 2000; Edwards et al., 2001). The dose-adjusted and transformed variables, $\log _{10}(\mathrm{BU})$ concentrations and $\log _{10}(\mathrm{HB})$ concentrations, are hereafter referred to as $\log B U$ and $\log H B$, respectively. $\log _{10}(\mathrm{HB} / \mathrm{BU})$ or $\log _{10}$ metabolic ratio is hereafter referred to as $\log \mathrm{MR}$ (metabolic ratio).

The primary aims of the study were achieved by model-based analysis of mean $\log \mathrm{MR}$ relative to $\log \mathrm{BU}$ concentrations and subject characteristics. The assumed linear statistical model for the logarithm of the metabolic ratio represented mean $\log \mathrm{MR}$ as a function of $\log \mathrm{BU}$ concentrations and other covariates representing cohort and genotype and other selected subject characteristics. This analysis of covariance (ANCOVA) model for logMR is linearly equivalent to an ANCOVA model for $\log \mathrm{HB}$ in which the regression equation includes the very same covariates: $\operatorname{logBU}$ concentrations, cohort, genotype, and other characteristics. By virtue of this linear equivalence, all results of the ANCOVA analysis are invariant regardless of whether the dependent variable is $\log \mathrm{HB}$ or $\log \mathrm{MR}$. We chose to focus on $\log \mathrm{MR}$ as the primary outcome variable for analysis. The analysis results presented would not be altered by using $\log \mathrm{HB}$ as the primary outcome variable.

To compare the three ethnic populations (AAW, caucasian, HAW) with respect to prevalence of variant genotypes, ethnicity-specific genotype frequencies were tabulated along with corresponding $95 \%$ confidence intervals. For each of five CYP2B6 polymorphisms (in intron 3, exon 4, exon 5, exon 7, exon 9), a Fisher's exact test procedure of size $\alpha=0.0125$ was used to test the null hypothesis "no differences among the three ethnic populations." The size of the test, $\alpha=0.0125=$ $0.05 / 4$ instead of $0.05 / 5$ (i.e., a Bonferroni adjustment for multiple comparisons) was used to account for the well described linkage disequilibrium between exon 4 and exon 5 and the almost perfect redundancy between these two alleles noted in Table 3. If at least one of the five $P$ values was smaller than $\alpha=0.0125$ then the overarching null hypothesis $\left(\mathrm{H}_{\mathrm{o}}\right)$ "no differences among the three ethnic populations with respect to genotype prevalence rates for these five genotypes" was to be rejected.

To characterize the relationship between $\log$ MR and ethnicity, sex, and genotype, an ANCOVA model was fitted in which the dependent variable was log $\mathrm{MR}$, and the independent variables included the covariate $\operatorname{logBU}$ concentrations centered at its mean $(1.83 \mathrm{ng} / \mathrm{ml})$, terms representing membership in the four cohorts, and terms representing genotype with respect to intron 3 , exon 5 , and exon 9. Due to differences in CYP2B6 haplotypes in different ethnic groups, the effects of $C Y P 2 B 6$ genetic variation may vary in these populations. Therefore we assumed that the effects of any variant genotype may not be the same from one ethnic group to another. Accordingly, the model also included interaction terms representing differential effects of intron 3 , exon 5 , and exon 9 across cohorts. It should be noted that the data did not support additional inclusion of exon 4, exon 7 , and exon 9 in the model: the values for exon 4 were highly redundant of those for exon 5 due to linkage disequilibrium (note: these two polymorphisms are commonly designated as the $C Y P 2 B 6^{*} 6$ allele), and the values for exon 7 and exon 9 varied little across subjects.

Fitting the primary ANCOVA model to the data provided statistical estimates of the parameters (i.e., regression coefficients). These statistical estimates were used to compute tests of hypotheses and to compute estimates of mean $\log$ MR at given levels of cohort, genotype (intron 3, exon 5, exon 9), and $\log B U$. Mean differences defining direct effects of cohort membership, and mean differences defining direct effects of genotype, as well as differences between mean differences that define genotype-by-cohort interaction effects also were estimated.

A second primary test was performed regarding genotype: to establish that intron 3, exon 5, and exon 9 have predictive value even when cohort membership is taken into account, an $F$-test procedure of size $\alpha=0.05$ was used to test the null hypothesis $\left(\mathrm{H}_{\mathrm{o}}\right)$ "intron 3 , exon 5 , and exon 9 have no predictive value." If, as expected, $\mathrm{H}_{\mathrm{o}}$ was rejected, then further tests of component subhypotheses were to be performed; e.g., $H_{o}$ (1) "intron 3 has no predictive value," etc. Similarly, a secondary hypothesis test was performed, designed to detect the existence of any association between $\log \mathrm{MR}$ and cohort membership that remained after having accounted for genotype. If this overall $\mathrm{H}_{\mathrm{o}}$ was rejected, then further tests of component subhypotheses were to be performed (HAW versus AAW, etc.). This hierarchical hypothesis testing strategy was designed to control the overall rates of type I error while optimizing statistical power (Muller and Stewart 2006).

In support of the primary analyses, we performed conventional diagnostic computations (e.g., analysis of residuals) with graphical and tabular summaries of the results. We also performed a number of auxiliary analyses to evaluate the robustness of the primary results to perturbations of the assumptions and methods. The auxiliary analyses included fitting the primary model with the following: exon 4 replacing exon 5 in the regression equation, omitting the genotype-by-subpopulation interactions, and several other analyses.

Several secondary and exploratory analyses also were performed as part of the comprehensive examination of the data. As an aid to interpretation of primary results, the genotype variables were excluded from the primary model and a secondary hypothesis $\left(\mathrm{H}_{0}\right)$, "the sex/ethnicity subpopulation differences are zero," was tested to demonstrate the existence of sex/ethnicity differences when genotype was not taken into account. The predictive value of age and BMI or weight was explored by modifying the primary model to include additional covariates in the model. Additional covariates considered were centered at their sample means [(age-27), (BMI-26), (weight-72), (height-167), (timing of blood draw-6)], and terms representing them were included in the regression equation. Finally, as an aid to the interpretation of the primary results, an auxiliary analysis was performed to examine the relationship between $\log \mathrm{BU}$ concentrations and ethnicity, sex, and genotype. For this ANCOVA model the dependent variable was $\log B U$ concentrations and the independent variables were the same as in the primary analyses.

All statistical computations were performed using SAS System software (version 9.2; SAS Institute Inc., Cary, NC). The fitting of the linear regression models reported relied on algorithms provided by mixed linear model procedure and an $F$-test procedure of size $\alpha=0.05$ was used to test hypotheses. Confidence intervals for variance parameter estimators were obtained via mixed linear models procedure. Influence diagnostics (e.g., Cook's Distance, the PRESS statistic, etc.) were computed using linear regression model procedure. In sensitivity analyses, auxiliary robust regression methods were implemented using nonlinear model procedure.

\section{Results}

Descriptive Tabulations. The four cohorts were similar in age. The men were taller, on average, than the women. Average weight was greatest for the AAW and CAM cohorts. Weights across the four 
cohorts ranged from 44 to $130 \mathrm{~kg}$. The CAW cohort had the lowest average BMI, whereas the AAW cohort had the highest average BMI (Table 1).

Blood samples used were withdrawn 6 hours postdose. At 6-hour time point mean sampling times and standard deviations were: $6.01 \pm$ 0.41 hours; $6.03 \pm 0.94$ hours; $6.03 \pm 0.64$ hours, and $6.03 \pm 0.12$ hours for CAM, CAW, AAW, and HAW, respectively. Geometric means with corresponding $95 \%$ confidence intervals are shown in Table 2 for dose-adjusted BU and HB concentrations at 6 hours postdose and for the calculated MR. Whereas the mean BU concentration for Hispanic women was lower compared with the other three cohorts, mean HB concentrations were similar that resulted in an approximately $50 \%$ higher MR for Hispanic women.

Association between CYP2B6 Genotype Frequency and Race/ Ethnicity. Table 3 depicts the distribution of the genotypes of the major functional CYP2B6 polymorphisms for each of the four cohorts. Associations between ethnicity and frequency for any of the five studied genotypes were not detected. Although there appeared to be differences in variant allelic frequency among ethnic groups for intron 3 , we were unable to conclude these differences are real based on a prespecified adjustment for multiple genotype comparisons.

Dependence of CYP2B6 Activity on Race/Ethnicity, Sex, and CYP2B6 Polymorphisms. The primary analysis used an ANCOVA model for $\log M R$ to evaluate the predictive value of $\operatorname{logBU}$ concentrations, sex, ethnicity, genotypes (intron 3, exon 5, exon 9) and genotype-by-sex/ethnicity interactions. Because exon 4 and exon 5 polymorphisms are in almost complete linkage disequilibrium, only exon 5 was included in the first analysis to save degrees of freedom and preserve power. Within the range of log-BU concentrations experienced by the subjects, the fitted model indicated that $\log \mathrm{HB}$ concentration increases by 0.13 units for every one-unit increase in $\log$ BU concentration $(P<0.0001)$. Association of $\log$ MR with sex and ethnicity was not detected $(P=0.7325)$. In contrast, the null hypothesis that the five genotypes have no predictive value was rejected $(P=0.0033)$. Occurrence of variant intron $3(P=0.0013)$ or exon $5(P=0.0017)$ was associated with lower activity compared with subjects with wild-type intron 3 and exon 5. Association with exon 9 was not detected $(P=0.7415)$. The null hypothesis that the magnitudes of the genotype effects are the same for all three ethnic populations was not rejected; i.e., the genotype-by-ethnicity interaction terms in the model were not statistically significant $(P=$ $0.3275)$.

Table 4 illustrate estimates of mean $\log M R$ for the four sex/ ethnicity cohorts for four genotype profiles: wild type for all three CYP2B6 genotypes, variant only in intron 3 , variant only in exon 5 , or

TABLE 1

Descriptive tabulations for age and body size $(N=100)$

Only descriptive statistics were performed on these data. Values are expressed as mean \pm S.D. The potential influence of these demographic variables in the analysis was addressed by considering them as covariates.

\begin{tabular}{lcccc}
\hline & $\begin{array}{c}\text { CAM } \\
(n=25)\end{array}$ & $\begin{array}{c}\text { CAW } \\
(n=25)\end{array}$ & $\begin{array}{c}\text { AAW } \\
(n=25)\end{array}$ & $\begin{array}{c}\text { HAW } \\
(n=25)\end{array}$ \\
\hline Age (years) & $25.7 \pm 7.5$ & $27.6 \pm 7.4$ & $27.6 \pm 7.6$ & $26.2 \pm 8.2$ \\
Range & $18-46$ & $18-41$ & $18-49$ & $19-44$ \\
Height $(\mathrm{cm})$ & $178.7 \pm 11.0$ & $164.5 \pm 5.8$ & $164.4 \pm 4.9$ & $161.4 \pm 5.6$ \\
Range & $159.5-211.0$ & $152.0-176.4$ & $156.0-173.0$ & $149.3-172.1$ \\
Weight $(\mathrm{kg})$ & $80.3 \pm 8.3$ & $60.6 \pm 9.1$ & $83.1 \pm 22.4$ & $63.2 \pm 8.9$ \\
Range & $65.0-99.3$ & $44.4-80.5$ & $52.8-129.7$ & $44.5-81.6$ \\
BMI $\left(\mathrm{kg} / \mathrm{cm}^{2}\right)$ & $25.4 \pm 4.0$ & $22.3 \pm 2.7$ & $30.9 \pm 8.5$ & $24.3 \pm 3.4$ \\
Range & $17.5-39.0$ & $18.1-29.9$ & $20.1-51.4$ & $19.1-30.9$ \\
\hline
\end{tabular}

AAW, African-American women; BMI, body mass index; CAM, Caucasian-American men; CAW, Caucasian-American women; HAW, Hispanic-American women. variant only in exon 9. $\mathrm{HB} / \mathrm{BU}$ metabolic ratio was much greater in Hispanic women, as 16 out of 26 HAWs had a variant intron 3 , and this variant is associated with lower metabolism $(P<0.0148)$.

When exon 4 was included in the regression equation in place of exon 5 the results were identical using either exon 5 or exon 4, which is expected because the two polymorphisms are in almost complete linkage disequilibrium. Two subjects had extremely low logMR values. Neither a deletion of these two subjects nor the use of robust regression methods provided a better fit to the data or led to a different interpretation of the results. Finally, inclusion of age and BMI in the primary model produced results that were fully consistent with those obtained via the primary model.

Relationship between Bupropion Concentrations and Subject Characteristics. A supportive analysis with $\log B U$ concentrations as the dependent variable was performed. The null hypothesis $\left(\mathrm{H}_{0}\right)$ that cohort effects and genotype-by-cohort interactions are all zero was rejected $(P=0.0243)$. Hierarchical testing of component subhypotheses detected ethnicity differences, with Hispanic Americans having a lower mean $\log \mathrm{BU}$ concentration than African Americans and Caucasian Americans. No differences between African Americans and Caucasian Americans were detected. An additional test designed to detect the existence of genotype differences not accounted for by sex/ ethnicity cohort membership also was not statistically significant.

\section{Discussion}

BU is an antidepressant and in humans is metabolized to three major metabolites: erythrohydrobupropion, threohydrobupropion, and $\mathrm{HB}$. The hydroxylation of $\mathrm{BU}$ to $\mathrm{HB}$ has been validated in vitro (Faucette et al., 2000) as a specific measure of CYP2B6 activity in liver microsomes. However, as opposed to in vitro incubations with microsomal preparations, clinical investigations are potentially complicated by competing metabolic pathways, as well as by renal and/or biliary elimination (Welch et al., 1987). Such alternative pathways of elimination may confound the relationship between $C Y P 2 B 6$ polymorphisms and CYP2B6 activity in the liver as measured by the in vivo clearance of BU. The extent to which carbonyl reduction of BU to erythrohydrobupropion and threohydrobupropion competes with CYP2B6mediated hydroxylation of BU in the liver is not known.

The purpose of this study was to identify important covariates that are associated with interindividual variation in CYP2B6 activity using the metabolism ratio of $\mathrm{BU}$ to $\mathrm{HB}$ as a surrogate measure. Knowledge of such covariates would further inform investigations intended to validate $\mathrm{BU}$ as a useful clinical probe. We hypothesized that specified CYP2B6 polymorphisms would account for any sex or ethnic diferences in $\mathrm{BU}$ that might be observed. In this study, there was no association of sex or ethnicity with CYP2B6 activity as measured by the HB/BU MR. However, an association was seen between CYP2B6 activity and polymorphisms in exon 5 and intron 3 . In addition, the results also suggest that the effect of the variant alleles on CYP2B6 activity was similar for both sexes and all ethnic groups examined.

The current study is the first to use bupropion metabolic ratio at a single time point to develop a model for evaluating CYP2B6 activity. A traditional dense concentration-time method of pharmacokinetic data analysis for this study would be preferable but would require upwards of 10 to 12 concentration-time samples per patient and would be clinically prohibitive. Therefore, a single 6-hour time point was selected for calculation of MR, guided in part by data from a previous study conducted by our research group. Using this approach, we found that the exon 5 allelic variant of $C Y P 2 B 6$, as well as the intron 3 variant, was independently associated with lower metabolic ratio compared with subjects with wild-type exon 5. The association of the 
TABLE 2

Bupropion and hydroxybupropion 6-hour concentrations $(\mathrm{ng} / \mathrm{ml})$ and metabolic ratio across sex/ethnicity cohorts $(N=100)$

Values are expressed as geometric means $( \pm 95 \%$ confidence intervals). As statistical analysis is more appropriately performed using analysis of covariance (ANCOVA) on log-transformed data, these tabulations are provided only for descriptive purposes. Each group had 25 patients.

\begin{tabular}{lcccccc}
\hline & \multicolumn{3}{c}{ Unadjusted } & & \multicolumn{2}{c}{ Dose-Adjusted $^{a}$} \\
\cline { 2 - 3 } & BU & HB & & BU & HB \\
\hline CAM & $66.67(57.99-76.65)$ & $260.95(206.40-329.92)$ & & $76.10(66.19-87.50)$ & $297.88(235.44-376.89)$ & $3.92(3.08,5.00)$ \\
CAW & $83.62(73.14-95.60)$ & $354.77(317.59-396.30)$ & & $71.56(63.82-80.25)$ & $303.61(271.80-339.14)$ & $4.24(3.62,4.97)$ \\
AAW & $66.13(53.67-81.49)$ & $258.55(191.14-349.73)$ & & $75.92(61.43-93.84)$ & $296.81(224.46-392.49)$ & $3.97(2.80,5.64)$ \\
HAW & $55.60(43.89-70.44)$ & $330.90(282.86-387.10)$ & & $49.74(38.48-64.31)$ & $296.05(256.49-341.71)$ & $6.01(4.60,7.85)$ \\
\hline
\end{tabular}

AAW, African-American women; BU, bupropion; CAM, Caucasian-American men; CAW, Caucasian-American women; HAW, Hispanic-American women; HB, hydroxybupropion.

${ }^{a}$ Prior to computation of the geometric mean, plasma concentrations were adjusted to correspond to a standard dose for a 70-kg weight individual.

exon 5 variant with lower metabolic ratio and our approach are supported by the recent report by Chung et al. (2011) where a metabolic ratio, calculated as the ratio of $\mathrm{HB} \mathrm{AUC}_{0-36}$ hours to $\mathrm{BU}$ $\mathrm{AUC}_{0-36 \text { hours, }}$ was lower in $C Y P 2 B 6^{*} 6$ (this nomenclature denotes the linked exon 4 and exon 5 variants) carriers than in $C Y P 2 B 6 * 6$ noncarriers among 35 healthy volunteers administered the same BU product at the same dose as used in our study. However, the small sample size per group, the absence of male group for each ethnicity, the reliance on the limited pharmacokinetic data based on 6-hour sampling time, the influence of alternative routes of elimination, and the extent of secondary metabolism of HB that has been described (Chung et al., 2011) should be considered as factors that potentially limit the findings of this study.

Women's livers have been reported to have higher amounts of CYP2B6 mRNA, protein, and activity than men's livers, and a $C Y P 2 B 6$ genotype-phenotype association was observed in livers obtained from women (Lamba et al., 2003). However, these previous investigations used human liver microsomes and the biotransformation of $S$-mephenytoin to nirvanol as a measure of CYP2B6 activity. We found no sex differences in bupropion metabolic ratio in our log-scale model-based analysis, although our findings are limited to Caucasian men and women. Our data suggest that there may not be clinically significant sex differences in human CYP2B6 activity or our methods were not sensitive enough to detect a small difference. Ethnic differences in CYP2B6 activity have also been observed when a human liver microsome assay was used (Lamba et al., 2003; Parkinson et al., 2004). Samples obtained from Hispanic women had 3.6- and 5.0-fold higher CYP2B6 activity compared with liver microsomes taken from Caucasian women and African-American women, respectively (Lamba et al., 2003). In this in vivo study, ethnic differences were observed in bupropion concentrations, with Hispanic women having lower concentrations even after dose adjustment compared with Caucasian and African-American women. However, there were no statistically significant differences in the bupropion metabolic ratio using our primary model. As mentioned above, the reduction of bupropion by hepatic carbonyl reductase represents a competing pathway of metabolism and may account for our observations in Hispanic women. For example, higher rates of carbonyl reduction in Hispanic women might result in lower plasma bupropion concentrations without observing an effect on CYP2B6-mediated hydroxylation if Michaelis constants $\left(K_{\mathrm{m}}\right)$ for bupropion reduction are much greater than the $K_{\mathrm{m}}$ for BU hydroxylation. Such effects would increase variability in the BU MR, perhaps confounding our ability to detect differences. Similarly, differences in the disposition and retention of $\mathrm{BU}$ and $\mathrm{HB}$ in various tissue compartments (e.g., related to their partitioning in low BMI or high BMI subjects), reflecting differences in their physiochemical properties, and in the polymorphic drug transport processes involved in their uptake and elimination will also increase variability in the MR. In addition, slow release of BU in the gastrointestinal tract, associated with the sustained release BU

TABLE 3

Genotype frequencies by sex/ethnicity cohorts

CAM and CAW were pooled for this test. The variant allelic number represents heterozygous and homozygous subjects for the polymorphism.

\begin{tabular}{|c|c|c|c|c|c|c|c|}
\hline Genotypes & & $\begin{array}{c}\text { CAM } \\
(n=25)\end{array}$ & $\begin{array}{c}\text { CAW } \\
(n=25)\end{array}$ & $\begin{array}{c}\text { AAW } \\
(n=25)\end{array}$ & $\begin{array}{l}\text { HAW } \\
(n=25)\end{array}$ & $\begin{array}{c}\text { Total } \\
(N=100)\end{array}$ & $\begin{array}{c}P \text { Value } \\
\text { (Test of Association) }^{a}\end{array}$ \\
\hline \multirow[t]{3}{*}{ Intron 3} & Variant & 11 & 13 & 6 & 16 & 46 & $* 0.0148$ \\
\hline & Wild type & 14 & 12 & 19 & 9 & 54 & \\
\hline & VAF & $22 \%$ & $34 \%$ & $12 \%$ & $44 \%$ & $28 \%$ & \\
\hline \multirow[t]{3}{*}{ Exon 4} & Variant & 10 & 12 & 16 & 12 & 50 & 0.4029 \\
\hline & Wild type & 15 & 13 & 9 & 13 & 50 & \\
\hline & VAF & $24 \%$ & $28 \%$ & $34 \%$ & $28 \%$ & $28.5 \%$ & \\
\hline \multirow[t]{3}{*}{ Exon 5} & Variant & 11 & 13 & 16 & 12 & 52 & 0.1163 \\
\hline & Wild type & 14 & 12 & 9 & 13 & 48 & \\
\hline & VAF & $26 \%$ & $30 \%$ & $36 \%$ & $30 \%$ & $30.5 \%$ & \\
\hline \multirow[t]{3}{*}{ Exon 7} & Variant & 0 & 0 & 1 & 0 & 1 & 0.5000 \\
\hline & Wild type & 25 & 25 & 24 & 25 & 99 & \\
\hline & VAF & $0 \%$ & $0 \%$ & $2 \%$ & $0 \%$ & $0.05 \%$ & \\
\hline \multirow[t]{3}{*}{ Exon 9} & Variant & 5 & 5 & 2 & 3 & 15 & 0.4380 \\
\hline & Wild type & 20 & 20 & 23 & 22 & 85 & \\
\hline & VAF & $10 \%$ & $10 \%$ & $4 \%$ & $6 \%$ & $7.5 \%$ & \\
\hline
\end{tabular}

AAW, African-American women; CAM, Caucasian-American men; CAW, Caucasian-American women; HAW, Hispanic-American women; VAF, variant alleli frequency.

${ }^{a}$ Fisher's exact test of the null hypothesis of no association between genotype and ethnic group (AAW, HAW, caucasian). $* P$ value less than $\alpha=0.0125$ was considered statistically significant. 
TABLE 4

Effect of genotype variants across sex/ethnicity cohorts $(\mathrm{N}=100)$

Values are expressed as mean estimates of $\log 10(\mathrm{MR}) \pm(95 \%$ confidence intervals).

\begin{tabular}{lcccc}
\hline \multicolumn{1}{c}{ Genotype } & CAM $(n=25)$ & CAW $(n=25)$ & AAW $(n=25)$ & HAW $(n=25)$ \\
\hline Wild type (only) & $0.774(0.648-0.901)$ & $0.812(0.672-0.952)$ & $0.845(0.678-1.013)$ & $0.726(0.460-0.992)$ \\
Intron 3 (only) & $0.638(0.530-0.746)$ & $0.676(0.567-0.784)$ & $0.500(0.314-0.686)$ & $0.680(0.540-0.819)$ \\
Exon 5 (only) & $0.587(0.480-0.693)$ & $0.624(0.518-0.731)$ & $0.614(0.496-0.731)$ & $0.643(0.494-0.792)$ \\
Exon 9 (only) & $0.809(0.659-0.960)$ & $0.847(0.689-1.004)$ & $0.900(0.579-1.221)$ & $0.600(0.324-0.847)$ \\
\hline
\end{tabular}

AAW, African-American women; CAM, Caucasian-American men; CAW, Caucasian-American women; HAW, Hispanic-American women; MR, metabolic ratio.

formulation used in our study, would have a more rate-limiting effect on BU metabolism in individuals with high CYP2B6 activity compared with those with low activity. Similarly, the hydroxylation of $\mathrm{BU}$ is also catalyzed to a lesser extent by other highly polymorphic $\mathrm{P} 450$ s that vary in concentration in human liver, and these would have a greater contribution in individuals with low CYP2B6 activity. These other factors would tend to reduce the range in the MR observed in our study.

Association between the CYP2B6 genotypes and sex/ethnicity cohorts was not detected in this study. It had been previously conjectured that a possible reason for ethnic differences in CYP2B6 activity could be differences in genotypic frequency. It was thought that differences in allelic frequency could, in part, explain ethnic group differences observed in CYP2B6 liver microsomal activity. In previous studies, intron 3 was found to be associated with lower CYP2B6 expression in women, exon 9 was associated with the lowest level of CYP2B6 activity in women's livers, and exon 4 516G $>$ T was shown to disrupt an exonic splice enhancer (Lamba et al., 2003; Thorn et al., 2010). The exon $5785 \mathrm{~A}>\mathrm{G}$ is frequently present in combination with exon $4516 \mathrm{G}>\mathrm{T}$ in the $C Y P 2 B 6^{*} 6$ haplotype. This haplotype is associated with lower CYP2B6 protein expression and BU hydroxylation in liver microsomes (Thorn et al., 2010). In our study, we detected an association between these functional CYP2B6 genotypes and CYP2B6 activity. Subjects having at least one variant allele tended to have a lower BU metabolic ratio than those carrying wild-type alleles; however, our results suggest the association of these variant alleles with lower metabolism is similar across all ethnic groups examined.

Variability in CYP2B6 activity and expression may depend on the combination of polymorphisms present in an individual. Also different genotypic combinations (haplotypes) may have different effects in different ethnic groups depending on other factors such as the transcription factor constitutive androstane receptor, which reflects environmental effects such as other drugs. However, because no interactions were detected between genotype and ethnicity, we have no direct evidence to conclude that there are differences in genotypic effects across different ethnic groups.

By using HB/BU metabolic ratio as a measure of CYP2B6 activity, we were able to confirm the influence of genetic variants that may be responsible for altered CYP2B6 function. Alterations in CYP2B6 activity may lead to differences in systemic exposure of drugs metabolized by CYP2B6 and therefore result in variation in therapeutic and toxic responses to these drugs in certain populations. Our results from this preliminary study suggest that only $C Y P 2 B 6$ genotype has an association with CYP2B6 activity. These results are encouraging and may allow for bupropion to be used as a probe substrate across sex and ethnicities as a measure of CYP2B6 activity. However, a formal prospective study that includes both men and women among all study groups and using a more optimized blood sampling time is needed to develop a robust predictive model for future clinical use.

\section{Acknowledgments}

The authors thank Dr. Colleen R.M. Lemmon for help with preparation of this manuscript, and Drs. Tedi A. Soule and Christine Walko for help in executing the study, as well as staff of the GCRC and McClendon UNC Hospital Laboratory's for assistance. The authors also thank the participants in this study. The authors thank Dr. Jatinder Lamba and Jeremy Hunt for expert technical assistance with $C Y P 2 B 6$ genotyping. Finally, the authors thank Dr. Sarah J. Schrieber who confirmed the correlation of the 6-hour time point with $\mathrm{BU}$ and $\mathrm{HB}$ using the original data from the study by Hogeland et al. (2007).

\section{Authorship Contributions}

Participated in research design: Lindley, Schuetz, Ilic, Chen.

Collected data and conducted experiments: Ilic, Thirumaran.

Performed data analysis: Hull, Ilic, Kashuba, Stewart.

Wrote or contributed to the writing of the manuscript: Ilic, Hawke, Hull, Stewart, Schuetz, Chen.

\section{References}

Chang TK, Weber GF, Crespi CL, and Waxman DJ (1993) Differential activation of cyclophosphamide and ifosphamide by cytochromes P-450 2B and 3A in human liver microsomes. Cancer Res 53:5629-5637.

Chung JY, Cho JY, Lim HS, Kim JR, Yu KS, Lim KS, Shin SG, and Jang IJ (2011) Effects of pregnane X receptor (NR1I2) and CYP2B6 genetic polymorphisms on the induction of bupropion hydroxylation by rifampin. Drug Metab Dispos 39:92-97.

Ding X and Kaminsky LS (2003) Human extrahepatic cytochromes P450: function in xenobiotic metabolism and tissue-selective chemical toxicity in the respiratory and gastrointestinal tracts. Annu Rev Pharmacol Toxicol 43:149-173.

Edwards LJ, Stewart PW, Muller KE, and Helms RW (2001) Linear equality constants in the general liver mixed model. Biometrics 57:1185-1190.

Ekins S, Vandenbranden M, Ring BJ, Gillespie JS, Yang TJ, Gelboin HV, and Wrighton SA (1998) Further characterization of the expression in liver and catalytic activity of CYP2B6. J Pharmacol Exp Ther 286:1253-1259.

Ekins S and Wrighton SA (1999) The role of CYP2B6 in human xenobiotic metabolism. Drug Metab Rev 31:719-754.

Erickson DA, Mather G, Trager WF, Levy RH, and Keirns JJ (1999) Characterization of the in vitro biotransformation of the HIV-1 reverse transcriptase inhibitor nevirapine by human hepatic cytochromes P-450. Drug Metab Dispos 27:1488-1495.

Faucette SR, Hawke RL, Lecluyse EL, Shord SS, Yan B, Laethem RM, and Lindley CM (2000) Validation of bupropion hydroxylation as a selective marker of human cytochrome P450 2B6 catalytic activity. Drug Metab Dispos 28:1222-1230.

Gervot L, Rochat B, Gautier JC, Bohnenstengel F, Kroemer H, de Berardinis V, Martin H, Beaune P, and de Waziers I (1999) Human CYP2B6: expression, inducibility and catalytic activities. Pharmacogenetics 9:295-306.

Gounden V, van Niekerk C, Snyman T, and George JA (2010) Presence of the CYP2B6 516G >T polymorphism, increased plasma efavirenz concentrations and early neuropsychiatric side effects in South African HIV-infected patients. AIDS Res Ther 7:1-9.

Hesse LM, Venkatakrishnan K, Court MH, von Moltke LL, Duan SX, Shader RI, and Greenblat DJ (2000) CYP2B6 mediates the in vitro hydroxylation of bupropion: potential drug interactions with other antidepressants. Drug Metab Dispos 28:1176-1183.

Hogeland GW, Swindells S, McNabb JC, Kashuba AD, Yee GC, and Lindley CM (2007) Lopinavir/ritonavir reduces bupropion plasma concentrations in healthy subjects. Clin Pharmacol Ther 81:69-75.

Hukkanen J, Mäntylä M, Kangas L, Wirta P, Hakkola J, Paakki P, Evisalmi S, Pelkonen O, and Raunio H (1998) Expression of cytochrome P450 genes encoding enzymes active in the metabolism of tamoxifen in human uterine endometrium. Pharmacol Toxicol 82:93-97.

Ingelman-Sundberg M, Sim SC, Gomez A, and Rodriguez-Antona C (2007) Influence of cytochrome P450 polymorphisms on drug therapies: pharmacogenetic, pharmacoepigenetic and clinical aspects. Pharmacol Ther 116:496-526.

Janmohamed A, Dolphin CT, Phillips IR, and Shephard EA (2001) Quantification and cellular localization of expression in human skin of genes encoding flavin-containing monooxygenases and cytochromes P450. Biochem Pharmacol 62:777-786.

Julious SA and Debarnot AM (2000) Why are pharmacokinetic data summarized by arithmetic mean? J Biopharm Stat 10:55-71. 
Kharasch ED, Mitchell D, and Coles R (2008) Stereoselective bupropion hydroxylation as an in vivo phenotypic probe for cytochrome P4502B6 (CYP2B6) activity. J Clin Pharmacol 48 464-474.

Kirchheiner J, Klein C, Meineke I, Sasse J, Zanger UM, Mürdter TE, Roots I, and Brockmöller J (2003) Bupropion and 4-OH-bupropion pharmacokinetics in relation to genetic polymorphisms in CYP2B6. Pharmacogenetics 13:619-626.

Lamba V, Lamba J, Yasuda K, Strom S, Davila J, Hancock ML, Fackenthal JD, Rogan PK, Ring B, and Wrighton SA, et al. (2003) Hepatic CYP2B6 expression: gender and ethnic differences and relationship to CYP2B6 genotype and CAR (constitutive androstane receptor) expression. J Pharmacol Exp Ther 307:906-922.

Muller KE and Stewart PW (2006) Linear Model Theory: Univariate, Multivariate, and Mixed Models, pp 289-310, Wiley Interscience, NY.

Nickerson DA, Tobe VO, and Taylor SL (1997) PolyPhred: automating the detection and genotyping of single nucleotide substitutions using fluorescence-based resequencing. Nucleic Acids Res 25:2745-2751.

Parkinson A, Mudra DR, Johnson C, Dwyer A, and Carroll KM (2004) The effects of gender, age, ethnicity, and liver cirrhosis on cytochrome P450 enzyme activity in human liver microsomes and inducibility in cultured human hepatocytes. Toxicol Appl Pharmacol 199 193-209.

Roy P, Yu LJ, Crespi CL, and Waxman DJ (1999) Development of a substrate-activity based approach to identify the major human liver P-450 catalysts of cyclophosphamide and ifosfamide activation based on cDNA-expressed activities and liver microsomal P-450 profiles. Drug Metab Dispos 27:655-666.
Streetman DS, Bertino JS, Jr, and Nafziger AN (2000) Phenotyping of drug-metabolizing enzymes in adults: a review of in-vivo cytochrome P450 phenotyping probes. Pharmacogenetics 10:187-216. Stresser DM and Kupfer D (1999) Monospecific antipeptide antibody to cytochrome P-450 2B6. Drug Metab Dispos 27:517-525.

Thorn CF, Lamba JK, Lamba V, Klein TE, and Altman RB (2010) PharmGKB summary: very important pharmacogene information for CYP2B6. Pharmacogenet Genomics 20:520-523.

Thum $\mathrm{T}$ and Borlak J (2000) Gene expression in distinct regions of the heart. Lancet $\mathbf{3 5 5}$ 979-983.

Tucker GT, Rostami-Hodjegan A, and Jackson PR (1998) Determination of drug-metabolizing enzyme activity in vivo: pharmacokinetic and statistical issues. Xenobiotica 28:1255-1273.

Ward BA, Gorski JC, Jones DR, Hall SD, Flockhart DA, and Desta Z (2003) The cytochrome P450 2B6 (CYP2B6) is the main catalyst of efavirenz primary and secondary metabolism: implication for HIV/AIDS therapy and utility of efavirenz as a substrate marker of CYP2B6 catalytic activity. J Pharmacol Exp Ther 306:287-300.

Welch RM, Lai AA, and Schroeder DH (1987) Pharmacological significance of the species differences in bupropion metabolism. Xenobiotica 17:287-298.

Address correspondence to: Roy L. Hawke, Division of Pharmacotherapy and Experimental Therapeutics, UNC Eshelman School of Pharmacy, CB \#7569, Kerr Hall Rm 3310, Chapel Hill, NC 27599-7360. E-mail: rhawke@email.unc.edu 